\title{
Elastic tubes: the ideal equipment for telehealth exercise medicine in the management of prostate cancer?
}

\author{
Georgios Mavropalias $^{1,2}$ (D)
}

Received: 1 December 2021 / Accepted: 21 January 2022 / Published online: 1 February 2022

(c) The Author(s) 2022

\begin{abstract}
Prostate cancer (PCa) affects 1 in 8 men, but exercise therapy has been shown to be a very effective intervention not only to induce physiological benefits but to also reduce the side effects of cancer treatments typically administered during PCa. The COVID19 pandemic has restricted access to exercise clinics, a problem which always existed for people living in rural and remote areas. This caused many exercise physiologists and researchers to transition their clinic-based exercise to online, home-based exercise. We would like to propose that researchers and exercise physiologists should consider the use of elastic tubes in both research and the clinical management of $\mathrm{PCa}$, when exercise programs are administered remotely, as their characteristics make them an ideal exercise equipment. In this article, the characteristics, considerations, and information on quantifying exercise dosage when using elastic tubes in remote exercise delivery are discussed.
\end{abstract}

Keywords Exercise medicine $\cdot$ Telehealth $\cdot$ Home-based exercise $\cdot$ Exercise therapy $\cdot$ Prostate cancer treatment

\section{Clinic-based to home-based exercise medicine for prostate cancer}

According to the American Cancer Society and Cancer Council Australia, 1 in 8 men in the USA and 1 in 6 men in Australia will be diagnosed with prostate cancer (PCa) during his lifetime. Exercise therapy has been shown to be a very effective intervention not only to induce physiological benefits but to also reduce the side effects of cancer treatments typically administered during PCa. Specifically, we have previously shown that resistance exercise training (RET), performed 2-3 times per week for 12 weeks, prevented muscle loss in PCa patients undergoing androgendeprivation therapy, even when combined with radiotherapy [1]. Moreover, 12 weeks of RET enhances the expression of serum myokines with tumor-suppressive effects in $\mathrm{PCa}$ patients [2]. Therefore, RET is an important intervention

Georgios Mavropalias

georgios.mavropalias@gmail.com

1 Exercise Medicine Research Institute, Edith Cowan University, 270 Joondalup Drive, Joondalup, WA 6027, Australia

2 School of Medical and Health Sciences, Edith Cowan University, Joondalup, Australia that can both exercise tumor-suppressive effects and also reduce the side effects of $\mathrm{PCa}$ treatments.

In the past 2 years, the COVID19 pandemic rendered a lot of people around the world unable to access exercise facilities, including PCa patients from receiving exercise therapy in exercise physiology clinics. In addition, the same challenge always existed with PCa patients who lived in remote or rural areas with no access to exercise physiology clinics. In the past year, many exercise physiologists and researchers transitioned their clinic-based RET to online, home-based RET. For example, in a recent paper, Winters-Stone et al. reported that they are conducting a home-based RET trial in PCa patients to investigate if it results in a fall reduction, by providing the participants with free weights to use in their homes [3]. We would like to propose that researchers and exercise physiologists should instead consider the use of elastic tubes in both research and the clinical management of $\mathrm{PCa}$, when exercise programs are administered remotely.

\section{The value of elastic tubes as a telehealth tool}

An elastic tube set is composed of different elastic tubes with metallic attachments at their ends, where grip handles or foot straps can be connected (Fig. 1). Commercial sets also contain door anchor points, to enable the performance 


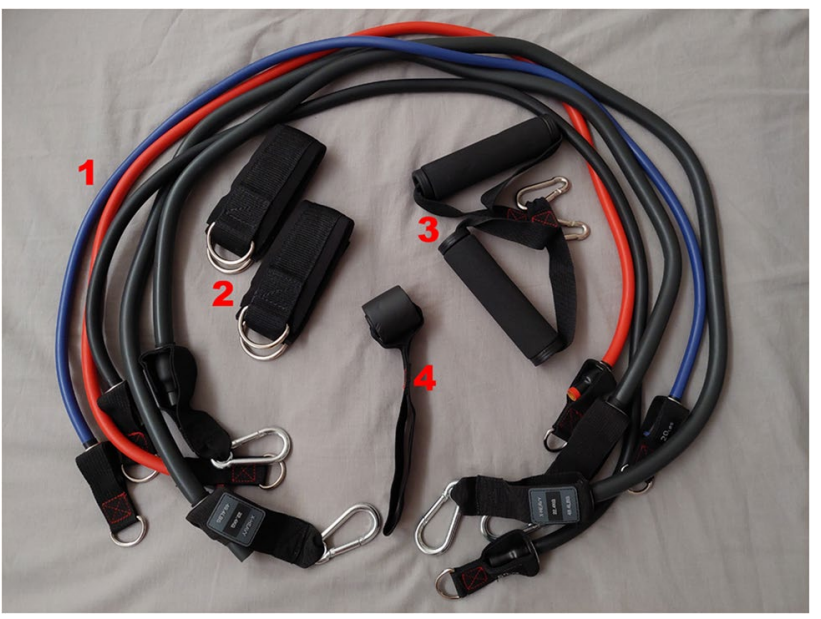

Fig. 1 An elastic tube set. 1: The elastic tubes of different resistance levels, 2: ankle straps, 3: handles, and 4: door anchor. Each of these attachment points can be connected with multiple tubes at one time, to increase resistance as required

of pulling exercises with two limbs. When multiple tubes are combined, they can result in loads of more than $70 \mathrm{~kg}$, with multiple handles and foot straps to allow the execution of almost every exercise that is possible with gym machine equipment. Moreover, when considering the different characteristics of resistance exercise equipment, elastic tubes can be considered an ideal equipment for telehealth exercise therapy. Specifically, the equipment scores very high in terms of compactness, home usage, adjustability, portability, simplicity, multiplicity, noiselessness, light weightlessness, inexpensiveness, effectiveness, safety, durability, and not requiring supervision [4].

\section{Elastic tubes in prostate cancer telehealth}

Exercise training using elastic tubes can effectively increase fat-free mass and muscle strength and decrease fat mass and frailty, to the same extent as free weights or machine equipment [5-9]. These findings are particularly important for PCa patients, which are typically over 65 years old, are sedentary, and in many cases frail [10]. In patients with PCa, frailty is associated with worse health-related quality of life [10], while higher muscle mass, and lower fat mass are associated with overall survival [11], and thus, exercise training with elastic tubes cannot only improve fitness, but can also result in crucial disease-specific benefits.

Transitioning from free weights or machine equipment can be confusing for the patients, as the resistance of the tubes is variable, and they cannot always perfectly approximate the load that would be explicitly indicated on free weights or machines. Regardless, one study in sedentary middle-aged women successfully used perceived exertion in the active muscles, increasing fat-free mass and strength to the same extent as weight machines [8]. Thus, we recommend using perceived exertion or effort, two concepts which can be easily understood even by patients unaccustomed to exercise terms and quantification of exercise dosage. PCa patients can be provided with elastic tubes, either for clinical practice or research, along with a guidebook, either printed or online, demonstrating the correct usage of the equipment, the available exercises, and a scale of perceived effort. In addition, the exercise routine can also be supplemented with bodyweight exercises.

Quantifying exercise dosage correctly is important to tolerability and adherence to exercise in people with cancer [12]. The patient can be prescribed specific sets and repetitions, and asked to attach different tubes for each exercise, to achieve a target perceived effort. The patient could then write down the total load from the combination of the tubes used, so that the dosage can be quantified. The length of the band greatly affects the elastic force exerted; therefore, the patient should be told to keep their distance from the attachment point constant. Moreover, the patient can also report if there were any myoskeletal pains, and the exercise program can be modified accordingly.

The field of telehealth exercise medicine for PCa is still emerging, and there is a lot of potential to improve the delivery of exercise therapy to PCa patients who have limited access to exercise physiology services. We urge researchers and exercise physiologists to consider the use of elastic tubes in both research and the clinical management of $\mathrm{PCa}$, as an inexpensive, portable, safe, and effective exercise equipment.

Author contribution GM conceptualized and designed this commentary, wrote, reviewed, and revised the manuscript. The author approved the final manuscript as submitted and agreed to be accountable for all aspects of the work.

Funding Open Access funding enabled and organized by CAUL and its Member Institutions.

Code availability Microsoft Word

\section{Declarations}

Ethics approval N/A

Consent to participate N/A

Consent for publication N/A

Conflict of interest The authors declare no competing interests.

Open Access This article is licensed under a Creative Commons Attribution 4.0 International License, which permits use, sharing, adaptation, distribution and reproduction in any medium or format, as long 
as you give appropriate credit to the original author(s) and the source, provide a link to the Creative Commons licence, and indicate if changes were made. The images or other third party material in this article are included in the article's Creative Commons licence, unless indicated otherwise in a credit line to the material. If material is not included in the article's Creative Commons licence and your intended use is not permitted by statutory regulation or exceeds the permitted use, you will need to obtain permission directly from the copyright holder. To view a copy of this licence, visit http://creativecommons.org/licenses/by/4.0/.

\section{References}

1. Newton RU, Mavropalias G, Fragala MS et al (2021) Radiotherapy before or during androgen-deprivation therapy does not blunt the exercise-induced body composition protective effects in prostate cancer patients: a secondary analysis of two randomized controlled trials. Exp Gerontol 151:111427. https://doi.org/10. 1016/j.exger.2021.111427

2. Kim J-S, Wilson RL, Taaffe DR et al (2021) Myokine expression and tumor-suppressive effect of serum following 12 weeks of exercise in prostate cancer patients on ADT. Med Sci Sports Exerc. https://doi.org/10.1249/MSS.0000000000002783

3. Winters-Stone KM, Boisvert C, Li F et al (2021) Delivering exercise medicine to cancer survivors: has COVID-19 shifted the landscape for how and who can be reached with supervised group exercise? Support Care Cancer. https://doi.org/10.1007/ s00520-021-06669-w

4. Nuzzo JL (2021) Content analysis of patent applications for strength training equipment filed in the United States before 1980. J Strength Cond Res 35:2952-2962. https://doi.org/10.1519/JSC. 0000000000004116

5. Daryanti Saragih I, Yang Y-P, Saragih IS et al (2021) Effects of resistance bands exercise for frail older adults: a systematic review and meta-analysis of randomised controlled studies. J Clin Nurs. https://doi.org/10.1111/jocn.15950

6. McGinley SK, Armstrong MJ, Boulé NG, Sigal RJ (2015) Effects of exercise training using resistance bands on glycaemic control and strength in type 2 diabetes mellitus: a meta-analysis of randomised controlled trials. Acta Diabetol 52:221-230. https://doi. org/10.1007/s00592-014-0594-y

7. Folkins E, Sahni S, Ryan J et al (2021) Concentric and eccentric force changes with elastic band and isotonic heavy resistance training: a randomized controlled trial. Int J Sports. Phys Ther 16:756-765. https://doi.org/10.26603/001c.23672

8. Colado JC, Triplett NT (2008) Effects of a short-term resistance program using elastic bands versus weight machines for sedentary middle-aged women. J Strength Cond Res 22:1441-1448. https:// doi.org/10.1519/JSC.0b013e31817ae67a

9. Turban C, Culas C, Deley G (2014) Effects of a short-term resistance program using elastic bands or weight machines in cardiac rehabilitation. Sci Sports 29:143-149. https://doi.org/10.1016/J. SCISPO.2013.07.005

10. Hamaya T, Hatakeyama S, Momota M et al (2021) Association between the baseline frailty and quality of life in patients with prostate cancer (FRAQ-PC study). Int J Clin Oncol 26:199-206. https://doi.org/10.1007/s10147-020-01798-4

11. Lopez P, Newton RU, Taaffe DR et al (2021) Associations of fat and muscle mass with overall survival in men with prostate cancer: a systematic review with meta-analysis. Prostate Cancer Prostatic Dis. https://doi.org/10.1038/s41391-021-00442-0

12. Fairman CM, Nilsen TS, Newton RU et al (2020) Reporting of resistance training dose, adherence, and tolerance in exercise oncology. Med Sci Sports Exerc 52:315-322. https://doi.org/10. 1249/MSS.0000000000002127

Publisher's note Springer Nature remains neutral with regard to jurisdictional claims in published maps and institutional affiliations. 